\title{
Optical behavior of GRB 061121 around its X-Ray shallow decay phase
}

\author{
T. Uehara ${ }^{1}$, M. Uemura ${ }^{2}$, A. Arai $^{3}$, R. Yamazaki ${ }^{4}$, K. S. Kawabata ${ }^{2}$, M. Ohno ${ }^{5}$, Y. Fukazawa ${ }^{1}$, T. Ohsugi ${ }^{2}$, \\ M. Yoshida ${ }^{2}$, S. Sato ${ }^{6}$, and M. Kino ${ }^{6}$ \\ ${ }^{1}$ Department of Physical Science, Hiroshima University, 1-3-1 Kagamiyama, Higashi-Hiroshima 739-8526, Japan \\ e-mail: uehara@hep01.hepl.hiroshima-u.ac.jp \\ 2 Hiroshima Astrophysical Science Center, Hiroshima University, 1-3-1 Kagamiyama, Higashi-Hiroshima 739-8526, Japan \\ 3 Faculty of Science, Kyoto Sangyo University, Motoyama, Kamigamo, Kita-Ku, Kyoto-City 603-8555, Japan \\ ${ }^{4}$ Department of Physics and Mathematics, Aoyama Gakuin University, 5-10-1 Fuchinobe, Sagamihara 252-5258, Japan \\ 5 Institute of Space and Astronautial Science, Japan Aaerospace Exploration Agency, 3-1-1 Yoshinodai, Chuo-ku Sagamihara, \\ Kanagawa 252-5120, Japan \\ ${ }^{6}$ Department of Physics, Nagoya University, Furo-cho, Chikusa-ku, Nagoya 464-8602, Japan
}

Received 12 October 2010 / Accepted 5 November 2010

\begin{abstract}
Aims. We report on a detailed study of the optical afterglow of GRB 061121 with our original time-series photometric data. Along with our optical observations and public X-ray data, we discuss the origin of its optical and X-ray afterglows.

Methods. We observed the optical afterglow of Swift burst GRB 061121 with the Kanata 1.5-m telescope at Higashi-Hiroshima Observatory. Our observation covers a period just after an X-ray plateau phase. We also performed deep imaging with the Subaru telescope in 2010 in order to estimate the contamination by the host galaxy.

Results. In the light curve, we find that the optical afterglow also exhibited a break as in the X-ray afterglow. However, our observation suggests a possible hump structure or a flattening period before the optical break in the light curve. There is no sign of such a hump in the X-ray light curve.

Conclusions. This implies that the optical emitting region was distinct from the X-ray one. The hump in the optical light curve was possibly caused by a passage with the typical frequency of synchrotron emission from another forward shock distinct from the early afterglow. The observed decay and spectral indices are inconsistent with the standard synchrotron-shock model. As a results, the observation requires a change in microphysical parameters in the shock region or prior activity by the central engine. Alternatively, the emission during the shallow decay phase may be a composition of two forward shock emissions, as indicated by the hump structure in the light curve.
\end{abstract}

Key words. gamma-ray burst: general

\section{Introduction}

Gamma-ray bursts (GRBs) and their afterglows are widely believed to be emission from relativistically expanding shells (e.g., Zhang \& Mészáros 2004; Mészáros 2006). GRBs, or prompt emissions are considered to arise from internal shocks caused by collisions between the shells. After the collisions, the shell keeps expanding and generates an external that shock collides with the interstellar medium. As a result, synchrotron emission from the shocked region is observed as afterglows. This synchrotronshock model successfully reproduces the observed temporal evolution of the spectral energy distributions (SEDs) of late afterglows. According to this model, the flux of the synchrotron emission from afterglows is described in a power-law form, that is, $f_{v}(t) \propto t^{-\alpha} v^{-\beta}$, where $\alpha$ and $\beta$ are a decay index and a spectral slope, respectively (Sari et al. 1998). A jet geometry has been suggested by an achromatic break observed in the light curves of afterglows (e.g., Rhoads 1997; Sari et al. 1999). This has recently been called into question because chromatic breaks were also detected when the jet scenario predicted achromatic ones (Willingale et al. 2007).

Owing to quick identifications and notifications of GRBs by the Swift satellite, the number of early afterglows being observed has been increasing in all wavelengths (Gehrels et al. 2004). $\mathrm{X}$-ray light curves of early afterglows, in particular, turned out to have more complicate profiles than those previously expected from the standard synchrotron-shock model. Although a simple power-law decay was expected in the standard model, the early X-ray light curves actually consist of three stages with different decay indices: the initial steep decay ( $\alpha \sim 3-5)$, the shallow decay ( $\alpha \sim 0.5-1.0$ ), and the normal decay phases ( $\alpha \sim 1$ ) (Nousek et al. 2006; O'Brien et al. 2006). While the steep decay phase is probably a high-latitude emission of the prompt emission (Kumar \& Panaitescu 2000; Yamazaki et al. 2006; Liang et al. 2006; Zhang et al. 2006), the origin of the shallow decay phase is currently unknown. Several models have been proposed for this phase, for example, the late energy injection into the shocked region, the time-dependent microphysics in the shock, or prior outflow emission (Sari \& Meszaros 2000; Nousek et al. 2006; Zhang \& Mészáros 2001; Panaitescu et al. 2006; Toma et al. 2006; Ioka et al. 2006; Dado et al. 2006; Uhm \& Beloborodov 2007; Yamazaki 2009).

Each of those models predicts distinct behaviors of SED variations in early afterglows. Simultaneous multiwavelength observations are required to provide crucial clues to the nature of the early afterglow phase. Panaitescu et al. (2006) report that 


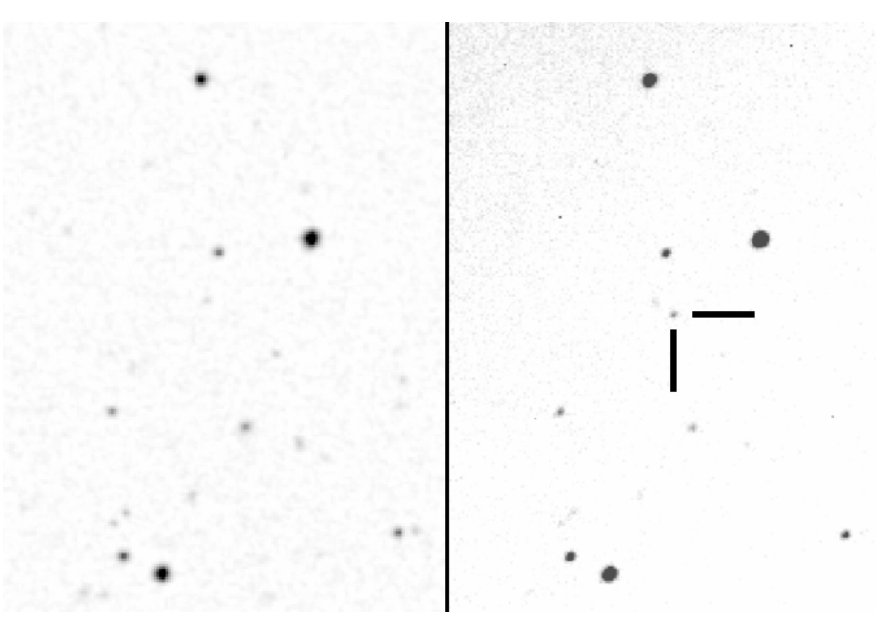

Fig. 1. Optical images of the field of GRB 061121 in POSS2 (left panel) and observed with the Kanata 1.5-m telescope (right panel). The field of view is $4^{\prime} \times 3^{\prime}$ and the top is north. The afterglow is an object marked with the black bars in the right panel.

the early break after the shallow decay phase is chromatic on the basis of 6 afterglows. Yost et al. (2007) additionally report that GRB 051109A also exhibits a clear chromatic break. Panaitescu et al. (2006) propose that the observed light curves require the temporal evolution of microphysical parameters in the emitting region of early and late afterglows. On the other hand, some afterglows apparently exhibit achromatic breaks after the X-ray shallow decay phase. Krühler et al. (2009) report on optical-IR and X-ray light curves of GRB 080710, in which an achromatic break was observed. Blustin et al. (2006) report another example of a possible achromatic break in GRB 050525A, while X-ray flares make it difficult to accurately determine a break time (also see, Klotz et al. 2005). In some of past cases, optical observations were too sparse to determine break times and to capturethe detailed behavior on either side of the breaks. We definitely need new observations in which break times can be determined accurately both in X-ray and optical light curves.

GRB 061121 was detected by the Swift Burst Alert Telescope (BAT) at 15:22:29 (UT) 21 November 2006 (Page et al. 2006). Swift also reported the discovery of a bright optical afterglow with Ultraviolet/Optical Telescope (UVOT), which was soon confirmed at $14.9 \mathrm{mag}^{1}$ by ground-based telescopes (Yost et al. 2006). Its redshift was estimated to be $z=1.314$ by spectroscopic observations of the optical afterglow (Bloom et al. 2006). This bright burst is a typical GRB following a well-known empirical relationship between $E_{\mathrm{p}}$ and $E_{\text {iso }}$ (Amati et al. 2006). The early X-ray light curve has several breaks like those in other systems observed in the Swift era (Nousek et al. 2006). Prompt onsets of multiwavelengths observations for GRB 061121 provided a unique opportunity to study the temporal evolution of X-ray and optical afterglows (Yost et al. 2006; Melandri et al. 2006; Uemura et al. 2006). Page et al. (2007) report on multiwavelength data during the prompt and afterglow phases of this GRB. According to them, both X-ray and optical flux monotonically decayed, which can be described with an early exponential rise followed by a power-law decay phase.

Here we report on our optical and infrared observations using the Kanata 1.5-m telescope. Our continuous time-series observations enabled us to reveal the optical behavior near the X-ray shallow decay phase. We describe the details of our observations in Sect. 2. Combined with other published data, we report the temporal evolution of the optical and X-ray afterglows in

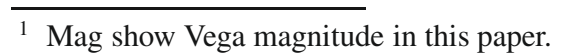

Sect. 3. In Sect. 4, we discuss the nature of the variations in the light curves using the synchrotron-shock model. Finally, we summarize our results in Sect. 5.

\section{Observation and data analysis}

\subsection{Optical observations}

Our observation started at 16:37 (UT) 2006 November 21, $4.6 \times 10^{3}$ s after the GRB trigger time, and ended at 19:57 (UT). The observation was performed with TRISPEC attached to the Kanata 1.5-m telescope at Higashi-Hiroshima Observatory of Hiroshima University. TRISPEC is a simultaneous imager and spectrograph with polarimetry covering both optical and nearinfrared wavelengths (Watanabe et al. 2005). We used the imaging mode for to observe GRB 061121 and obtained 77 sets of $R_{\mathrm{c}}$, $J$, and $K_{\mathrm{s}}$ band images. The exposure time of an $R_{\mathrm{c}}$-band image was $123 \mathrm{~s}$. During the $123 \mathrm{~s}$ exposure, short exposures of a few seconds were taken for NIR arrays, and yielded net exposures of 120 and 96 s for each $J$ - and $K_{\mathrm{s}}$-band image, respectively.

The central wavelength of the TRISPEC's $R_{\mathrm{c}}$ system is $\sim 620 \mathrm{~nm}$, slightly shifted from the standard one $(=645 \mathrm{~nm})$. The difference in magnitude between these systems is expected to be 0.008 mag when a power-law spectrum with a spectral index of 1.0 is assumed. In the following discussion, we neglect this small difference.

We show an example of the obtained images in the right panel of Fig. 1. We also show the same field in the Second Palomer Sky Survey (POSS2) in the left hand panel for comparison. After making dark-subtracted and flat-fielded images, we obtained magnitudes of the afterglow and comparison stars using a Java-based PSF photometry package. For a comparison star, we used USNO-B1.0 0768-0239968 $\left(\mathrm{RA}=9^{\mathrm{h}} 48^{\mathrm{m}} 54^{\mathrm{s}} .78\right.$, Dec $\left.=-13^{\circ} 1^{\prime} 17^{\prime \prime} .9 ; R_{\mathrm{c}}=18.02\right)$. The comparison star was constant within 0.02 mag during our observation, checked by USNO-B1.0 0767-0229365 $\left(\mathrm{RA}=09^{\mathrm{h}} 49^{\mathrm{m}} 05^{\mathrm{s}} .080\right.$, Dec $=$ $\left.-13^{\circ} 13^{\prime} 22^{\prime \prime} .21\right)$. Using neighboring USNO stars, we checked systematic errors of magnitudes that depend on comparison stars, and found that it is smaller than $0.2 \mathrm{mag}$. The comparison star is the same as used in Halpern et al. (2006a), Halpern \& Armstrong (2006a), and Halpern \& Armstrong (2006b), which present observations in a late stage of the afterglow. In the following section, we analyzed of our light curve in conjunction with those late-time observations. 
T. Uehara et al.: Optical behavior of GRB 061121 around its X-Ray shallow decay phase

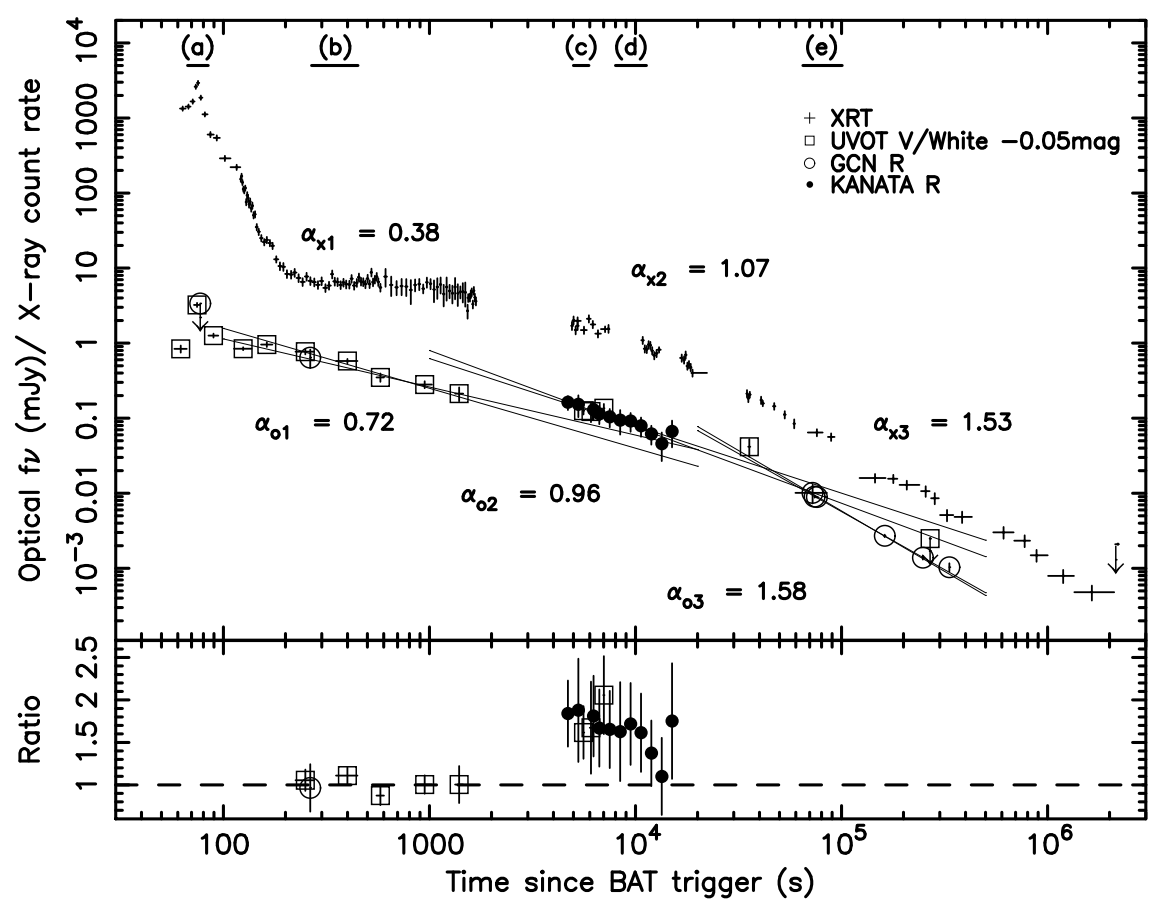

Fig. 2. Optical and X-ray light curves of GRB 061121 afterglows. The abscissa denote the time since the GRB trigger in seconds. In the top panel, the ordinate denotes the flux density in mJy for optical data and the count rate for X-ray data observed with XRT. Our observations and X-ray observations by XRT are indicated by the filled circles and crosses, respectively. Open circles and squares are optical observations with $R_{\mathrm{c}}$ and $V /$ White light (UVOT) bands reported to GCN or taken by UVOT, respectively. The solid lines are 95\% confidence regions of the best-fitted power-law models for optical light curves. The labels, a), b), c), d), and e) represent the time intervals for the SED analysis (see Sect. 3.2). In the bottom panel, the ordinate denotes the ratio of the observed flux density to the best-fitted model of the plateau phase. $V$ band points are shifted by -0.05 to match the $R_{\mathrm{c}}$ band points.

Table 1. Results of our photometric observation.

\begin{tabular}{rcccc}
\hline \hline Time $(\mathrm{s})^{*}$ & $R_{\mathrm{c}}{ }^{* *} \mathrm{mag}$ & $R_{\mathrm{c}}^{\prime} \mathrm{mag}^{\dagger}$ & error & $N^{\ddagger}$ \\
\hline 4699 & 18.16 & 18.17 & 0.03 & 3 \\
5283 & 18.23 & 18.24 & 0.15 & 4 \\
6261 & 18.40 & 18.42 & 0.08 & 2 \\
6675 & 18.53 & 18.55 & 0.09 & 5 \\
7501 & 18.63 & 18.66 & 0.09 & 6 \\
8427 & 18.74 & 18.76 & 0.18 & 7 \\
9466 & 18.77 & 18.80 & 0.10 & 8 \\
10632 & 18.92 & 18.95 & 0.10 & 9 \\
11941 & 19.18 & 19.22 & 0.09 & 10 \\
13409 & 19.49 & 19.55 & 0.23 & 11 \\
15056 & 19.10 & 19.14 & 0.21 & 12 \\
\hline
\end{tabular}

Notes. ${ }^{(*)}$ Time since the GRB trigger. ${ }^{(* *)}$ Raw $R_{\mathrm{c}}$ magnitude. ${ }^{(\dagger)} R_{\mathrm{c}}$

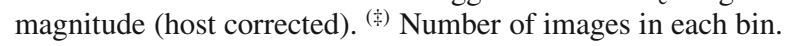

Additionally, Table 1 contains $R_{\mathrm{c}}$-magnitudes obtained by our optical observations. In this table, the magnitudes are averages in equally spaced bins in the logarithmic scale of the time. While we obtained $J$ and $K_{\mathrm{s}}$ band images using TRISPEC, IR afterglows were not significantly detected. Typical 3-sigma upper limits of each frame are $16.0 \mathrm{mag}$ in the $J$ band and 13.7 in $K_{\mathrm{s}}$.

For deep photometry of the host galaxy component, we obtained $R_{\mathrm{c}}$-band images with the 8.2-m Subaru Telescope and the Faint Object Camera and Spectrograph (FOCAS; Kashikawa et al. 2002) on 2010 May 7 (UT). The total exposure time was $240 \mathrm{~s}$. We can easily recognize the host galaxy as a point source at the GRB afterglow position in this image. Using the same comparison star as mentioned above, we derived the magnitude of the host galaxy to be $R_{\mathrm{c}}=22.99 \pm 0.03$.

\subsection{Data analysis of the Swift data}

We analyzed the public data of GRB 061121 observed with X-Ray Telescope (XRT) and UVOT on Swift. We processed the XRT all orbits of data, adopting the standard screening with the XRT pipeline FTOOL xrtpipeline (Version: 0.10.3). We extracted light curve and spectra with a rectangular $40 \times 20$-pixel region for the windowed timing (WT) mode and 40-pixel radius region for the photon counting (PC) mode from the source position. The background was also extracted from $40 \times 20$-pixel region for the WT mode, and 40-pixel radius region for the PC mode, far from the source. While beginning the GRB for WT mode data and PC mode data, we found that the count rate is high enough to cause the pile-up effect, and we adopted the standard pile-up correction as described by Romano et al. (2006) and Vaughan et al. (2006).

In the following section, the unit of time is set to be seconds from the GRB trigger. Optical and X-ray parameters are indicated by subscripts of "O" and "X", respectively.

\section{Results}

\subsection{Optical and $X$-ray light curves}

We show the X-ray and optical light curves of GRB 061121 in Fig. 2 (Page et al. 2007; Yost et al. 2006; Melandri et al. 2006; Sonoda et al. 2006; Marshall et al. 2006; Golenetskii et al. 2006; Halpern et al. 2006b; Cenko 2006; Halpern et al. 2006a; Efimov et al. 2006a; Halpern \& Armstrong 2006a,b; 

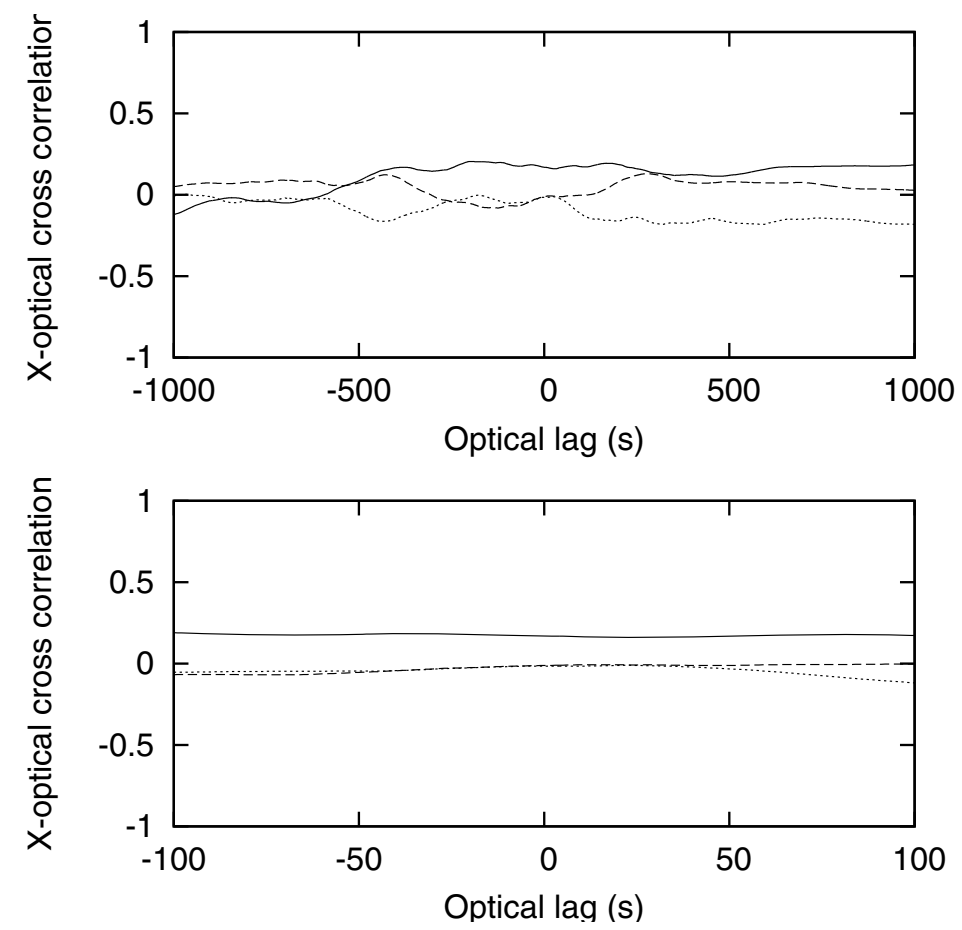

Fig. 3. X-ray/optical cross correlations for short-term variations. The solid, dashed, and dotted lines were calculated from the data of $4.8 \times 10^{3} \mathrm{~s}<$ $t<7.5 \times 10^{3} \mathrm{~s}, 1.0 \times 10^{4} \mathrm{~s}<t<1.4 \times 10^{4} \mathrm{~s}$, and $1.6 \times 10^{4} \mathrm{~s}<t<1.9 \times 10^{4} \mathrm{~s}$, respectively. The upper and lower panels show correlation functions on long and short time scales. No significant correlation can be seen.

Efimov et al. 2006b). The contribution of the host galaxy is subtracted from the optical flux. The flux density of the host galaxy is $2.14 \mu \mathrm{Jy}$ in $R_{\mathrm{c}}$, which was estimated based on our Subaru observation as described in Sect. 2. The flux density was corrected for the Galactic extinction of $E(B-V)=0.04$ (Schlegel et al. 1998). The absolute magnitude of the host galaxy is $-21.86 \mathrm{mag}$ in $R_{\mathrm{c}}$.

According to Page et al. (2007), the X-ray light curve of GRB 061121 is divided into 4 phases depending on their decay indices. In this paper, we follow their definition of the phases for the X-ray light curves, that is, an initial flare, a plateau $\left(\alpha_{\mathrm{X} 1}=0.38 \pm 0.06\right)$, a shallow decay $\left(\alpha_{\mathrm{X} 2}=1.07 \pm 0.05\right)$, and a normal decay phase $\left(\alpha_{\mathrm{X} 3}=1.53 \pm 0.03\right)$. The errors of these parameters, as well as other parameters given in this paper, represent $1-\sigma$.

In the optical light curve, we can see a possible flare in a very early phase at $t=76 \mathrm{~s}$. This implies that the optical flux may be associated with the prompt emission in X-rays and $\gamma$-rays in this phase (Page et al. 2007). In the X-ray plateau phase, the optical light curve can be described with a simple power-law decay. Using $V$-band observations by UVOT from 240 to $2000 \mathrm{~s}$, we calculated the power-law decay index to be $\alpha_{\mathrm{O} 1}=0.72 \pm 0.08$.

During the subsequent shallow decay phase, our observation revealed a monotonic fading of the optical afterglow. The light curve can be described with a simple power-law having a decay index of $\alpha_{\mathrm{O} 2}=0.96 \pm 0.06$. Page et al. (2007) report a $V$-band decay index of $0.66 \pm 0.04$ from the onset of the fading to a break at $\sim 2.5 \times 10^{4} \mathrm{~s}$. This decay index was estimated based on the exponential-to-power-law model, which assumes a monotonic fading during the fading stage of the afterglow. The $\alpha_{\mathrm{O} 2}$ estimated from our time-series photometry is, however, significantly more than reported in Page et al. (2007). We tried to fit a simple power-law model to the optical light curve from $240 \mathrm{~s}$ to $16 \mathrm{ks}$ including our data. For the fitting, our $V$-band data was shifted to the corresponding $R_{\mathrm{c}}$-band magnitude. The $V-R_{\mathrm{c}}$ of the afterglow was estimated from two almost simultaneous $V$ and $R_{\mathrm{c}}$-band observations, $t \sim 280$ and $\sim 6300 \mathrm{~s}$. The average color of the afterglow is calculated as $V-R_{\mathrm{c}}=0.05$. The bestfitted parameters yield a chi-square/d.o.f of $76.3 / 15$. This value is too high to conclude that the afterglow decayed with a simple power-law form from $240 \mathrm{~s}$ to $16 \mathrm{ks}$, and instead suggests that there is a substructure around the termination of the X-ray plateau phase.

The optical light curve, then, exhibits another break around $3 \times 10^{4} \mathrm{~s}$, which is followed by the normal decay phase described with $\alpha_{\mathrm{O} 3}=1.58 \pm 0.03$. By fitting a broken power-law model, we calculated a break time of $4.6_{-2.3}^{+4.5} \times 10^{4} \mathrm{~s}$. No significant time lag is detected for the last break from the shallow decay to the normal decay phase between the X-ray and optical breaks, while the errors of break times are quite large; $3.2_{-0.6}^{+2.1} \times 10^{4} \mathrm{~s}$ for the X-ray and $4.6_{-2.3}^{+4.5} \times 10^{4} \mathrm{~s}$ for the optical break times. It is noteworthy that the optical decay index is almost the same as the X-ray one after this break. According to the standard synchrotron shock model, this strongly indicates a passage with the cooling frequency of the synchrotron emission within the optical band at the break time.

We searched possible correlations between X-ray and optical short-term variations. We calculated cross-correlations using 3 segments in which simultaneous optical and X-ray data are available. The optical data ware divided into the following parts; i) $4.8 \times 10^{3} \mathrm{~s}<t<7.5 \times 10^{3} \mathrm{~s}$, ii) $1.0 \times 10^{4} \mathrm{~s}<t<1.4 \times 10^{4} \mathrm{~s}$, and iii) $1.6 \times 10^{4} \mathrm{~s}<t<1.9 \times 10^{4} \mathrm{~s}$. The resulting cross-correlations are shown in Fig. 3. The correlation functions are flat and show no prominent feature. We cannot detect any significant correlations between optical and X-ray short-term variations.

\subsection{Spectral energy distribution}

Figure 4 shows infrared-X-ray SEDs. The figure contains 5 panels in which simultaneous optical and X-ray observations are 
T. Uehara et al.: Optical behavior of GRB 061121 around its X-Ray shallow decay phase

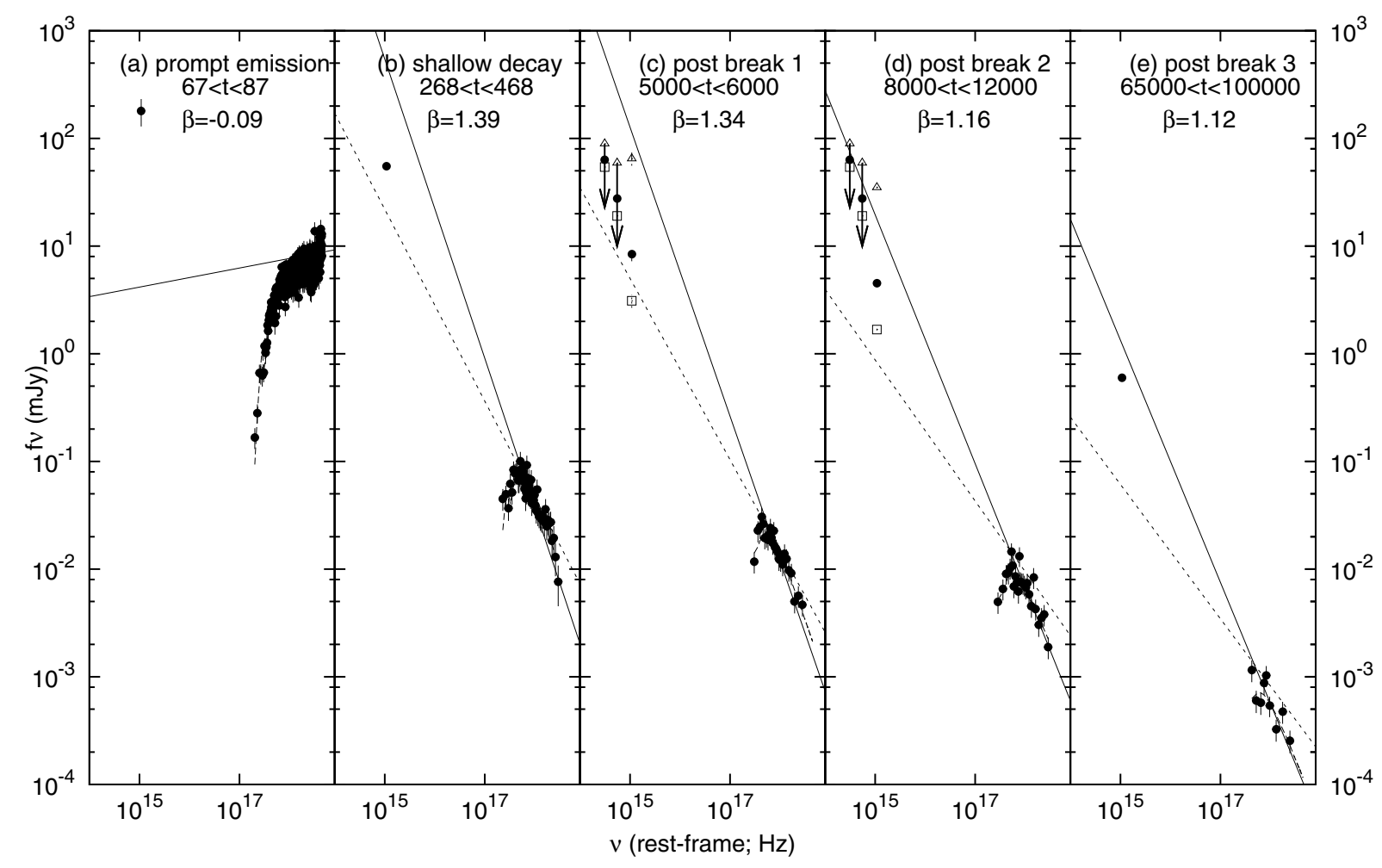

Fig. 4. Spectral energy distributions derived for the time intervals of a) $6.7 \times 10^{1} \mathrm{~s}<t<8.7 \times 10^{1} \mathrm{~s}$, b) $\left.4.3 \times 10^{2} \mathrm{~s}<t<5.4 \times 10^{2} \mathrm{~s}, \mathbf{c}\right)$ $5.0 \times 10^{3} \mathrm{~s}<t<6.0 \times 10^{3} \mathrm{~s}$, d) $8.0 \times 10^{3} \mathrm{~s}<t<1.2 \times 10^{4} \mathrm{~s}$, and e) $6.5 \times 10^{4} \mathrm{~s}<t<1.0 \times 10^{5} \mathrm{~s}$. The time intervals of the SEDs for each panel are indicated in figure 2. The abscissa and ordinate denote the rest frame frequency in $\mathrm{Hz}$ and the flux density in mJy, respectively. The filled circles are X-ray observations by XRT, optical observations reported to GCN, and our optical-IR observations. The IR observations just provide upper limits of the flux. For the extragalactic extinction, the optical and IR data were corrected with the "Q2" model (Maiolino et al. 2001). The open triangles and squares are data in which their corrections for the extinctions were performed with the Milky Way model and the "Q1" model, respectively (Guidorzi et al. 2007). We omit arrows indicating upper limits of the IR points of the open triangles and squares. The solid lines represent the best-fitted power-law model for the X-ray spectra. The dotted lines are expected spectral slopes from the synchrotron-shock model.

Table 2. best-fit parameters for X-ray spectra.

\begin{tabular}{ccc}
\hline \hline Time interval $(\mathrm{s})$ & $\beta$ & $\chi^{2} /$ d.o.f. \\
\hline $6.7 \times 10^{1}<t<8.7 \times 10^{1}$ & $0.09 \pm 0.01$ & $267 / 195$ \\
$4.3 \times 10^{2}<t<5.4 \times 10^{2}$ & $1.39 \pm 0.04$ & $19 / 17$ \\
$5.0 \times 10^{3}<t<6.0 \times 10^{3}$ & $1.34 \pm 0.05$ & $14 / 12$ \\
$8.0 \times 10^{3}<t<1.2 \times 10^{4}$ & $1.16 \pm 0.04$ & $10 / 9$ \\
$6.5 \times 10^{4}<t<1.0 \times 10^{5}$ & $1.12 \pm 0.09$ & $10 / 9$ \\
\hline
\end{tabular}

shown for the 5 phases. We fitted a power-law model with a single absorption component for the X-ray spectra. All 5 spectra in Fig. 4 can be described with absorption models with a hydrogen column density of $N_{\mathrm{H}}=2.2 \pm 0.15 \times 10^{21} \mathrm{~cm}^{-2}$ in the observer's frame, which corresponds to $N_{\mathrm{H}}=9.2 \times 10^{21} \mathrm{~cm}^{-2}$ in the rest frame. As can be seen from the figure, spectral slopes $\beta$ $\left(f_{v} \propto v^{-\beta}\right)$ were slightly larger in the plateau phase (panel b) and a phase just after the optical break (panel c) than those in later phases. Table 2 contains results of our best-fitted parameters in each period.

In the figure, the optical flux was corrected for the galactic and extragalactic extinctions. The extragalactic extinction was corrected with the relationship between the visual extinction $A_{\mathrm{V}}$ and the hydrogen column density $N_{\mathrm{H}}$ for the "Q2" model in Maiolino et al. (2001) $\left(N_{\mathrm{H}} / A_{\mathrm{V}}=3.3 \times 10^{21} \mathrm{~cm}^{-2}\right)$. We estimated the $N_{\mathrm{H}}$ from the best-fitted model of X-ray spectra, and set it to $N_{\mathrm{H}}=9.2 \times 10^{21} \mathrm{~cm}^{-2}$ as mentioned above. The conversion from $A_{\mathrm{V}}$ to those in other bands was performed following the equations in Cardelli et al. (1989).
For the correction of the extragalactic extinction, we used the "Q2" model because it provides the most plausible opticalIR SEDs, as shown below. According to the synchrotron-shock model, the spectral slope at the optical region should be $\beta_{\mathrm{O}}=$ $\beta_{\mathrm{X}}-0.5$ in the case of $v_{m}<v_{\mathrm{O}}<v_{\mathrm{c}}<v_{\mathrm{X}}$, where $v_{m}$ and $v_{\mathrm{c}}$ are typical and cooling frequencies of the synchrotron emission from a forward shock (Sari et al. 1998). In Fig. 4, we show the expected spectral slope of $\beta_{\mathrm{O}}=\beta_{\mathrm{X}}-0.5$ with $v_{\mathrm{c}}=10^{18} \mathrm{~Hz}$. The optical flux is required to be over this dotted lines in order to satisfy the condition expected by the synchrotron-shock model. In addition to the "Q2" model, the figure also contains opticalIR points corrected with the Milky Way model $\left(N_{\mathrm{H}} / A_{\mathrm{V}}=1.6 \times\right.$ $\left.10^{21} \mathrm{~cm}^{-2}\right)$ and with the "Q1" model $\left(N_{\mathrm{H}} / A_{\mathrm{V}}=6.7 \times 10^{21} \mathrm{~cm}^{-2}\right)$ (Maiolino et al. 2001). As can be seen in panel (c), a high $A_{\mathrm{V}}$ provided by the Milky Way model yields an unnaturally sharp break between $R_{\mathrm{c}}$ and $J$-bands. Corrected by the "Q1" model, the optical flux is too low to be interpreted by the synchrotronshock model with $\beta_{\mathrm{O}}=\beta_{\mathrm{X}}-0.5$. The models for SMC $\left(N_{\mathrm{H}} / A_{\mathrm{V}}=\right.$ $\left.1.5 \times 10^{22} \mathrm{~cm}^{-2}\right)$ and LMC $\left(N_{\mathrm{H}} / A_{\mathrm{V}}=7.6 \times 10^{21} \mathrm{~cm}^{-2}\right)$ also yield lower optical flux. Thus, the "Q2" model provides the best correction among those models.

Near the peak of the prompt emission, as can be seen in panel (a) of Fig. 4, the optical flux is further above the power-law component of X-rays. This indicates that the emission mechanism or source of the optical emission is distinct from those of the prompt X-ray and $\gamma$-ray emission.

In panel (c) of Fig. 4, there is a difference between the optical flux and the spectrum extrapolated from the X-ray data. The 
SED, therefore, requires a spectral break between the optical and $\mathrm{X}$-ray bands. This is consistent with the situation for the case of $v_{m}<v_{\mathrm{O}}<v_{\mathrm{c}}<v_{\mathrm{X}}$. In the standard model, $v_{\mathrm{c}}$ evolves with time, decreasing for a constant density medium, increasing for a wind medium. Since our findings privilege a constant density medium as discussed in Sect. 4.2, we expect at one point that $v_{\mathrm{c}}$, as it decreases with time, will cross the optical band. At that time, the optical and X-ray decay will become identical, and the SED will be compatible with a simple power law. This is the case in panel (e), suggesting that $v_{\mathrm{c}}$ crossed the optical band around $t=5 \times 10^{4} \mathrm{~s}$.

\section{Discussion}

\subsection{A possible hump structure in the optical light curve}

Here, we discuss the substructure in the optical light curve around $5 \mathrm{ks}$. The behavior of the optical and X-ray light curves is unclear in the transition phase from the plateau to the shallow decay phases $\left(1.5 \times 10^{3} \mathrm{~s} \lesssim t \lesssim 4.6 \times 10^{3} \mathrm{~s}\right)$. Fig. 2 shows regions of the power-law decay model for each phase of the optical afterglow with $95 \%$ confidence. As indicated by these lines, our observation suggests that the plateau phase is terminated by a flattening phase or a hump in the optical afterglow.

We checked the significance of this hump with a correction of colors, because the best-fitted models were calculated with data taken with different bands; the $V$ - and $R_{\mathrm{c}}$-band observations in the plateau and shallow decay phases, respectively. The lower panel of Fig. 2 shows the ratio of the observations to the bestfitted model of the plateau phase. We converted $V$-band observations to our $R_{\mathrm{c}}$-band ones, by adding $V-R_{\mathrm{c}}=0.05$ to the $V$-magnitudes. The hump appears over a 2.2-sigma level even with the color correction.

In contrast, there is no sign of such a hump in the X-ray light curve between those two phases. In addition, there is a large difference in the optical and X-ray decay indices in the plateau phase, compared with those in the subsequent shallow and normal decay phases. The X-ray and optical light curves apparently exhibit different behaviors during the X-ray plateau phase and the transition phase to the shallow decay phase.

\subsection{Implication to the synchrotron-shock model}

In this section, we discuss the behavior of the afterglow of GRB 061121 based on the synchrotron-shock model. In the case of $v_{m}<v_{\mathrm{O}}<v_{\mathrm{c}}<v_{\mathrm{X}}$, Urata et al. (2007) propose a relation between the decay indices of the X-ray and optical bands described as $\alpha_{\mathrm{X}}-\alpha_{\mathrm{O}}=1 / 4$. In the plateau and shallow decay phases of GRB 061121, the $\alpha_{\mathrm{X}}-\alpha_{\mathrm{O}}$ is $0.34 \pm 0.10$ and $0.11 \pm 0.08$, respectively. The classical synchrotron-shock model, thus, fails to reproduce the observed light curves of GRB 061121 in the shallow decay phase. It can only marginally reproduce the light curve in the plateau phase.

Panaitescu et al. (2006) generalized the formulae of the synchrotron-shock model by including the variations in the en$\operatorname{ergy}(E)$ in the blast wave, the energy ratio for electrons and the magnetic field $\left(\varepsilon_{i}\right.$ and $\left.\varepsilon_{B}\right)$, and the ambient medium $(n)$ in the following form; $E(>\Gamma) \propto \Gamma^{-e}, \varepsilon_{B} \propto \Gamma^{-b}, \varepsilon_{i} \propto \Gamma^{-i}$, and $n(r) \propto r^{-s}$. The decay indices of optical and X-ray light curves are calculated as in Eqs. (9) and (10) in Panaitescu et al. (2006). Using those formulae, we evaluate the presence of the energy injection $(e>0)$ or the time variations of microphysical parameters $(b \neq 0$ or $i \neq 0$ ) for GRB 061121 .
We first assume $s=0$, namely the uniform distribution of the interstellar medium. In the following examination, we calculate $p$ from $\beta$ in each time interval shown in Table 2 . For the plateau phase, assuming $b=0$ and $i=0$, we find that the optical and $\mathrm{X}$-ray light curves yield inconsistent $e$, that is, $e=1.32 \pm 0.24$ and $4.05 \pm 0.37$ calculated from $\alpha_{\mathrm{O} 1}$ and $\alpha_{\mathrm{X} 1}$, respectively. This inconsistency can be reconciled only when $s$ takes a narrow range of $s=1.22 \pm 0.01$ and $e$ takes an unnaturally high value $(e \sim 7)$. In the other case of $s>0$, the inconsistency in $e$ becomes more extreme. These results indicate that the observed light curves during the plateau phase cannot be reproduced only with the energy injection scenario. Temporal variations of the microphysical parameters are therefore required. Assuming $e=0$ and $s=0$, we obtain $b=-2.10 \pm 0.36$ and $i=2.04 \pm 0.12$. The positive $i$ implies a low efficiency of the energy for accelerating electrons in highly relativistic shocks.

During the shallow decay phase after the optical hump, the decay indices changed to $\alpha_{\mathrm{O} 2}=0.96$ and $\alpha_{\mathrm{X} 2}=1.07$. Even in this phase, the condition of $b=0$ and $i=0$ yields different $e$ values calculated from $\alpha_{\mathrm{O} 2}$ (yielding $e=0.64 \pm 0.20$ ) and $\alpha_{\mathrm{X} 2}$ $(e=1.19 \pm 0.25$ ). Assuming $e=0$, we obtain $b=-0.50 \pm 0.27$ and $i=0.75 \pm 0.12$ for this phase. It is interesting to note that the absolute values of both $b$ and $i$ decrease from the plateau to the shallow decay phases. From the early to late stages, the condition of the blast wave may resemble the classical picture in which no temporal variation in the microphysical parameters is required. Thus, the model proposed by Panaitescu et al. (2006) can explain the light curves in both the plateau and shallow decay phases by changing the microphysical parameters.

An alternative model was proposed by Ioka et al. (2006), who consider prior activities before the main prompt emission. According to their model, the shallow decay of X-ray afterglows appears because a blast wave obtains additional energy by colliding with prior ejecta without significant decelerating of shells. This model is possibly preferable for GRB 061121, since it has a precursor $\sim 75 \mathrm{~s}$ before the peak of the main prompt emission (Page et al. 2007). The precursor may be a sign of prior activity.

Ioka et al. (2006) define the the prior ejected mass as a power-law form of $\gamma$, that is, $M(<\gamma) \propto \gamma^{a}$. The decay indices of X-ray and optical afterglows are given with $\alpha_{\mathrm{X}}=(a-3) / 2+$ $(a-11)(p-2) / 8$ and $\alpha_{\mathrm{O}}=(7 a-25) / 8+(a-11)(p-2) / 8$ in the case of $v_{m}<v_{\mathrm{O}}<v_{\mathrm{c}}<v_{\mathrm{X}}$, respectively. For the plateau phase of GRB 061121, the X-ray and optical decay indices provide a consistent $a$ within errors for possible values of $p(2.2 \gtrsim p \gtrsim 2.8)$. In the case of $p=2.78$, for example, we obtained $a=4.94 \pm$ 0.48 from $\alpha_{\mathrm{X} 1}$ and $a=5.05 \pm 0.10$ from $\alpha_{\mathrm{O} 1}$. For the shallow decay phase, assuming $p=2.68$, for example, we calculated $a=5.99 \pm 0.44$ from $\alpha_{\mathrm{X} 2}$ and $a=5.23 \pm 0.10$ from $\alpha_{\mathrm{O} 2}$. Thus, the decay indices of the plateau and shallow decay phases can be reproduced with the prior activity model proposed by Ioka et al. (2006), while a temporal variation of $a$ would be needed.

Both models in Panaitescu et al. (2006) and Ioka et al. (2006) can only explain the observed light curves of GRB 061121 when time variations in $b, i$, or $a$ are allowed. The situation is more confusing when we consider the presence in the optical hump between the plateau and shallow decay phases. The discontinuity around the hump indicates a variation of the optical decay index, which means a further variation in $b, i$, or $a$ during the hump.

The hump in the optical light curve between the plateau and shallow decay phases is apparently not seen in the X-ray light curve. This implies that the dominant emitting regions are different in X-ray and optical afterglows around the hump. The hump structure reminds us of the two-component jet model; the hump may be explained with a scenario that the emission from 
a narrow jet may dominate in the plateau phase, while that from a wide jet became dominant after the hump (Sheth et al. 2003; Peng et al. 2005). In this case, the hump structure may appear when $v_{m}$ of the synchrotron emission from the wide jet passes the optical band. The classical synchrotron-shock model failed to reproduce the light curves possibly because of the composition of the two components in the plateau and shallow decay phases.

In the standard synchrotron-shock model, an increase in the density of the shock region would produce a hump in the optical light curve which is not seen in X-rays if $v_{\mathrm{c}}$ lies between the optical and X-ray bands (Panaitescu \& Kumar 2000). Hence, the optical hump of GRB 061121 may also be reproduced if the external shock passed through a high-density region in the interstellar medium at $t=2-6 \mathrm{ks}$.

We finally note that there are several sources that exhibit optical light curves analogous to GRB 061121, that is, GRB 021004 (Uemura et al. 2002), GRB 050525A (Klotz et al. 2005; Blustin et al. 2006), GRB 060117 (Jelínek et al. 2006), GRB 060526 (Dai et al. 2007), and GRB 061007 (Mundell et al. 2007). For all of them, an early decay phase was terminated by a flattening or a hump at $10^{2-4} \mathrm{~s}$, which was followed by a steeper decay phase. An important point is that the decay indices before and after the flattening phase are different. This characteristic feature may be observed commonly in a group of GRB afterglows. If the emission during the early decay phase has a different nature than during the later decay phase, the relationship of those two components possibly causes the diversity in light curves of optical afterglows and the correlation between early $\mathrm{X}$-ray and optical light curves.

\section{Summary}

We performed time-series photometry of the optical afterglow of GRB 061121 with the $1.5-\mathrm{m}$ Kanata telescope and reported on a detailed study of the afterglow with published X-ray and optical data. The decay index of the optical light curve was significantly different between the plateau and shallow decay phases. The optical light curve possibly has a hump structure between the plateau and shallow decay phases, while no sign of such a hump is seen in the X-ray light curve. The different behavior in the optical and X-ray light curves indicates that they have distinct emitting sources. The hump structure in the optical light curve may imply a passage with the typical frequency of the synchrotron emission from another forward shock that is distinct from the early afterglow. The optical decay index became the same as the X-ray one in the late phase after the final break at $\sim 4.6 \times 10^{4} \mathrm{~s}$. In conjunction with the temporal evolution of SEDs, we propose that this break is caused by the passage with the cooling frequency at the optical band. In both the plateau and shallow decay phases, the observed decay and spectral indices are inconsistent with the standard synchrotron-shock model. They requires a variation in the microphysical parameters in the shock region or prior activity by the central engine. It is also possible that they come from the composition of two forward shock components if the hump structure in the light curve was caused by another forward shock.

Acknowledgements. The authors are grateful to Dr. M. Watanabe for useful comments on the paper. This work was partly supported by a Grant-in-Aid from the Ministry of Education, Culture, Sports, Science, and Technology of Japan (17684004, 17340054, 19047004, 14079206, 18840032). Part of this work is supported by a Research Fellowship of Japan Society for the Promotion of Science for Young Scientists. We are also grateful to K. Maeda, T. Hattori and M. Tanaka for the opportunity of the Subaru observation for the host component. And, we are also grateful to Dr. Page, for useful UVOT data.

\section{References}

Amati, L., Frontera, F., Guidorzi, C., \& Montanari, E. 2006, GRB Coordinates Network, 5848, 1

Bloom, J. S., Perley, D. A., \& Chen, H. W. 2006, GRB Coordinates Network, 5826, 1

Blustin, A. J., Band, D., Barthelmy, S., et al. 2006, ApJ, 637, 901

Cardelli, J. A., Clayton, G. C., \& Mathis, J. S. 1989, ApJ, 345, 245

Cenko, S. B. 2006, GRB Coordinates Network, 5844, 1

Dado, S., Dar, A., \& De Rújula, A. 2006, ApJ, 646, L21

Dai, X., Halpern, J., Morgan, N., et al. 2007, ApJ 658, 509

Efimov, Y., Rumyantsev, V., \& Pozanenko, A. 2006a, GRB Coordinates Network, 5850, 1

Efimov, Y., Rumyantsev, V., \& Pozanenko, A. 2006b, GRB Coordinates Network, 5870, 1

Gehrels, N., Chincarini, G., Giommi, P., et al. 2004, ApJ, 611, 1005

Golenetskii, S., Aptekar, R., Mazets, E., et al. 2006, GRB Coordinates Network, 5837,1

Guidorzi, C., Gomboc, A., Kobayashi, S., et al. 2007, A\&A, 463, 539

Halpern, J. P., Mirabal, N., \& Armstrong, E. 2006b, GRB Coordinates Network, 5840,1

Halpern, J. P., Mirabal, N., \& Armstrong, E. 2006a, GRB Coordinates Network, 5847, 1

Halpern, J. P., \& Armstrong, E. 2006a, GRB Coordinates Network, 5851, 1 Halpern, J. P., \& Armstrong, E. 2006b, GRB Coordinates Network, 5853, 1

Ioka, K., Toma, K., Yamazaki, R., \& Nakamura, T. 2006, A\&A, 458, 7

Jelínek, M., Prouza, M., Kubánek, P., et al. 2006, A\&A, 454, L119

Kashikawa, N., Aoki, K., Asai, R., et al. 2002, PASJ, 54, 819

Klotz, A., Boer, M., Atteia, J. L., et al. 2005, A\&A, 439, L35

Krühler, T. Greiner, J., \& Afonso, P. 2009, A\&A, 508, 593

Kumar, P., \& Panaitescu, A. 2000, ApJ, 541, L51

Liang, E. W., Zhang, B., O’Brien, P. T., et al. 2006, ApJ, 646, 351

Maiolino, R., Marconi, A., \& Oliva, E. 2001, A\&A, 365, 37

Marshall, F. E., Holland, S. T., \& Page, K. L. 2006, GRB Coordinates Network, 5833,1

Melandri, A., Guidorzi, C., Mundell, C. G., et al. 2006, GRB Coordinates Network, 5827, 1

Mészáros, P. 2006, Reports Progr. Phys., 69, 2259

Mundell, C. G., Melandri, A., Guidorzi, C., et al. 2007, ApJ, 660, 489

Nousek, J. A., Kouveliotou, C., Grupe, D., et al. 2006, ApJ, 642, 389

O'Brien, P. T., Willingale, R., Osborne, J., et al. 2006, ApJ, 647, 1213

Page, K. L., Sakamoto, T., Marshall, F. E., et al. 2006, GCN Report, 15.1

Page, K. L., Willingale, R., Osborne, J. P., et al. 2007, ApJ, 663, 1125

Panaitescu, A. Kumar, P. 2000, ApJ, 543, 66

Panaitescu, A., Meszaros, P., Burrows, D., et al. 2006, MNRAS, 369, 2059

Peng, F., Konigel, A., \& Granot, J. 2005, ApJ, 626, 966

Piran, T. 1999, Phys. Rep., 314, 575

Rhoads, J. E. 1997, ApJ, 487, L1

Romano, P., Campana, S., Chincarini, G., et al. 2006, A\&A, 456, 917

Rykoff, E. S., Mangano, V., Yost, S. A., et al. 2006, ApJ, 638, L5

Sari, R., \& Piran, T. 1999, ApJ, 517, L109

Sari, R., \& Meszaros, P. 2000, ApJ, 535, L33

Sari, R., Piran, T., \& Narayan, R. 1998, ApJ, 497, L17

Sari, R., Piran, T., \& Halpern, J. P. 1999, ApJ, 519, L17

Schlegel, D. J., Finkbeiner, D. P., \& Davis, M. 1998, ApJ, 500, 525

Sheth, K. Frail, D. A., White, S., et al. 2003, ApJ, 595, L33

Sonoda, E., Maeno, S., Hara, T., et al. 2006, GRB Coordinates Network, 5830, 1

Toma, K., Ioka, K., Yamazaki, R., \& Nakamura, T. 2006, ApJ, 640, L139

Uemura, M., Kato, T., Ishioka, R., \& Yamaoka, H. 2002, PASJ, 55, L31

Uemura, M., Arai, A., \& Uehara, T. 2006, GRB Coordinates Network, 5828, 1

Uhm, Z. L., \& Beloborodov, A. M 2007, ApJ, 665, L93

Urata, Y., Yamazaki, R., Sakamoto T., \& Huang, K. 2007, ApJ, 668, L95

Vaughan, S., Goad, M. R., Beardmore, A. P., et al. 2006, ApJ, 638, 920

Watanabe, M., Nakaya, H., Yamamuro, Y., et al. 2005, PASP, 117, 870

Willingale, R., O’Brien, P. T., Osborne, J. P., et al. 2007, ApJ, 662, 1093

Yamazaki, R. 2009, ApJ, 690, L118

Yamazaki, R., Toma, K., Ioka, K., \& Nakamura, T. 2006, MNRAS, 369, 311

Yost, S. A., Schaefer, B. E., \& Yuan, F. 2006, GRB Coordinates Network, 5284, 1

Yost, S. A., Swan, H. F., Rykoff, E. S., et al. 2007, ApJ, 657, 925

Zhang, B., \& Mészáros, P. 2001, ApJ, 552, L35

Zhang, B., \& Mészáros, P. 2004, Int. J. Mod. Phys. A, 19, 2385

Zhang, B., Fan, Y. Z., Dyks, J., et al. 2006, ApJ, 642, 354 